\title{
Operations Militaires Contre Les Adf A Beni En Republique Democratique Du Congo :« Entre prestige et humiliation de l'Etat»
}

\author{
Par : Jaribu Muliwavyo ${ }^{1}$
}

\section{Introduction}

L'ampleur des massacres contre les populations civiles de Beni en République Démocratique du Congo (RDC) correspond au discours officiel du vendredi 24 Octobre 2014, lors du passage à Eringeti, de M. Richard Muyej Mangej, alors Ministre de l'Intérieur, Sécurité, Décentralisation et Affaires Coutumières, après le massacre de 84 civils par les ADF. Ce responsable national de la sécurité intérieure s'était exclamé en ce terme : « Je ne sais pas s'il y a des mots pour exprimer notre état d'âme. Ce qui est arrivé est grave, c'est un coup fort pour la nation. Je ne manquerai pas de dire que c'est une humiliation... ça fait la honte à la République... il nous faut absolument relever les défis ».Dans le même cadre de compassion aux victimes des massacres, le Nonce Apostolique Luis Mariano Montemayor s'est rendu à Mbelu, au quartier Rwangoma, commune Beu en ville de Beni où un autre massacre dont le bilan n'avait pas été révélée ${ }^{2}$, venait de se dérouler le 13 août 2016. Arrivé au lieu des massacres, il dénonce : «le silence honteux autour des tueries de Beni... ». Cet envoyé de Jorge Mario Bergoglio, Pape François, ajoute que : « Nous voulons voir concrètement comment faire vivre le travail de protection de la population... Il faut également la volonté politique du gouvernement de faire mieux...»».

L'on ne cesse de s'interroger pourquoi les vastes opérations militaires impliquant conjointement la Mission d'Observation des Nations Unies pour la Stabilisation du Congo (MONUSCO) et les Forces Armées de la République Démocratique du Congo (FARDC) mais quelques fois aussi les Forces pour la Défense du Peuple Ougandais (UPDF) ne parviennent pas à démanteler les bastions des rebelles Ougandais des Forces Alliées Démocratiques (ADF). Non seulement les populations locales sont en proie à des kidnappings et assassinats aux allures terroristes puisque tués à la hache et à la machette ; mais aussi les ADF et leurs ancêtres de la National Army for the Liberation of Uganda (NALU) ont imposé « une zone grise » ${ }^{3}$ sur un espace considérable du territoire nationale congolais depuis bientôt trente ans ${ }^{4}$. Cet exposé se veut être une critique systématique de la dynamique des opérations militaires déjà déployées dans la région de Beni pour éradiquer les ADF. Il s'intéresse aux interviews, aux déclarations et autres documents en rapport avec l'histoire immédiate du phénomène ADF.En plus de l'introduction et la conclusion, ce modeste travail s'articule sur trois points : la présentation critique de ces opérations, une brève discussion sur l'opération «Safisha Ruwenzori » et l'évaluation de l'opération « Sokola 1 ».

\section{Présentation critique des opérations militaires contre les ADF}

La présence des rebelles $\mathrm{ADF}$ dans la région du Ruwenzori où les conflits armés sont de plus en plus résiduels a déjà fait l'objet de plusieurs opérations militaires. Ces opérations sont listées dans le tableau :

Tableau 1. Récapitulation des opérations militaires dans le Ruwenzori

\begin{tabular}{|l|l|l|l|}
\hline OPERATIONS & OBJECTIFS & RESULTATS & FAIBLESSES \\
\cline { 3 - 4 } & & FORCES & -Création de la NALU; \\
1. Ruwenzori & Mater la poussée des rebelles du & -Reconquête de la région par les & -Pillages des biens de la population \\
1996 & $\begin{array}{l}\text { PLC/ et plus tard de la LDC qui } \\
\text { conquirent la quasi-totalité de la } \\
\text { région }\end{array}$ & $\begin{array}{l}\text { FAZ; } \\
\text {-Expulsion de quelques Chefs } \\
\text { rebelles ougandais ; } \\
\text {-Reddition de certains chefs }\end{array}$ & $\begin{array}{l}\text {-Extorsion et rançonnement } \\
\text {-Incendie de 462 maisons dans les }\end{array}$ \\
\hline
\end{tabular}

\footnotetext{
${ }^{1}$ Jaribu Muliwavyo est Chef de Travaux à la Faculté des sciences sociales administratives et politiques à l'Université de Goma. Il est en plus Député Provincial à l'Assemblée Législative du Nord-Kivu en République Démocratique du Congo.

${ }^{2}$ Le Gouvernement de la RDC parle de trente-six morts, la société civile évoque cinquante et une mort tandis que l'opposition parle d'une centaine des morts dont une grande partie jetée dans la rivière Semuliki.

${ }^{3}$ Les zones grises sont des espaces géographiques caractérisés par une insécurité récurrente et un déficit de contrôle étatique. Ce sont des régions instables investies par des organisations hors-la-loi. Pour plus d'éclaircissement, lire :

- Philippe Moreau Defarges.., « La gestion de zones grises », in Rams, Paris, 2003n pp59-70 ;

- Gaidz Minassian, Zones grises. Quand les Etats perdent le contrôle, éd. Autrement,

Paris, 2011, p17.

${ }^{4}$ Jaribu Muliwavyo, La dynamique du phénomène rebelle dans le Ruwenzori à la frontière Congolo-Ougandaise : Autopsie de la rébellion ADF-NALU, in Annales de L'Unigom, Vol V-Nº, Goma, Décembre 2014, pp.76-106
}

DOI: $10.9790 / 0661-1901015664 \quad$ www.iosrjournals.org $56 \mid$ Page




\begin{tabular}{|c|c|c|c|}
\hline & & $\begin{array}{l}\text { rebelles zaïrois avec leurs } \\
\text { troupes; } \\
\text {-Réinstallation des autorités } \\
\text { politico-administratives ; }\end{array}$ & $\begin{array}{l}\text { villages se situant entre Kasaka et } \\
\text { Museya; } \\
\text {-Braconnage organisé par les FAZ; } \\
\text {-Climat de méfiance entre les FAZ } \\
\text { et la population locale; } \\
\text {-Retranchement de quelques Chefs } \\
\text { rebelles en Ouganda; }\end{array}$ \\
\hline $\begin{array}{l}2 . \quad \text { Mountain } \\
\text { sweep (Balayer } \\
\text { la montagne) } \\
\text { Le } 27 \text { avril } \\
1998\end{array}$ & $\begin{array}{l}\text {-Sécuriser les frontières entre la } \\
\text { RDC et l'Ouganda ; } \\
\text {-Combattre la rébellion des } \\
\text { ADF }\end{array}$ & $\begin{array}{l}\text {-Ratissage de la région et } \\
\text { intégration au sein des FAC des } \\
\text { résidus des militaires des FAZ en } \\
\text { cachette }\end{array}$ & $\begin{array}{l}\text {-Cas d'enlèvements et assassinats } \\
\text { perpétrés par les ADF ; } \\
\text {-Incendie volontaire d'une grande } \\
\text { partie de la forêt naturelle du } \\
\text { Ruwenzori par l'UPDF et les FAC } \\
\text { à la recherche des ADF; } \\
\text {-Naissance d'une } \\
\text { rébellion : le RCD ; } \\
\text {-Ouverture de nouveaux maquis } \\
\text { vers le nord de la région de } \\
\text { Ruwenzori à Watalinga et à Beni- } \\
\text { Mbau dont Madina (1999). }\end{array}$ \\
\hline $\begin{array}{l}\text { 3. North Night } \\
\text { final (Dernière } \\
\text { nuit du Nord) } \\
\text { Le } 25 \text { décembre } \\
2005\end{array}$ & $\begin{array}{l}\text {-Pousser les ADF à adhérer aux } \\
\text { programmes DDRRR et } \\
\text { d'Amnistie ; } \\
\text {-traquer les combattants ADF } \\
\text { réfractaires à ces programmes. }\end{array}$ & $\begin{array}{l}\text {-Découvertes de plusieurs indices } \\
\text { de collaboration entre les ADF et } \\
\text { certains officiers tant des FARDC } \\
\text { que de l'UDPF } \\
\text {-Implication de la MONUSCO } \\
\text { dans la traque des ADF au moyen } \\
\text { de ses hélicoptères de combat; } \\
\text {-Documentation et publication } \\
\text { par les experts de l'ONU des } \\
\text { structures et organisation des } \\
\text { ADF. }\end{array}$ & $\begin{array}{l}\text {-Eparpillement des ADF dans les } \\
\text { villages et forêts en Secteur de } \\
\text { Beni-Mbau et en Chefferie des } \\
\text { Watalinga; } \\
\text {-Implication des ADF dans le } \\
\text { commerce frontalier de l'or, du } \\
\text { bois, du café et de la papaïne; } \\
\text {-Relâchement de la traque. }\end{array}$ \\
\hline $\begin{array}{l}\text { 4. Safisha } \\
\text { Ruwenzori } \\
\text { (Nettoyer le } \\
\text { Ruwenzori) } \\
\text { Le 28 juin 2010 }\end{array}$ & $\begin{array}{l}\text {-Eradiquer l'activisme des } \\
\text { ADF; } \\
\text {-Mettre en application des } \\
\text { Accords de Ngurundoto; } \\
\text {-Sceller l'amitié J.Kabila - Y. } \\
\text { Museveni avant la célébration } \\
\text { de la fête du cinquantenaire de } \\
\text { la RDC, le } 30 \text { juin } 2010\end{array}$ & $\begin{array}{l}\text {-Destruction du quartier général } \\
\text { des ADF à Mughalika ; } \\
\text {-Arrivée des délégations } \\
\text { gouvernementales en mission } \\
\text { d'encouragement aux FARDC et } \\
\text { de réconfort à la population de } \\
\text { Mutwanga; } \\
\text {-Installation des bases de la } \\
\text { MONUSCO à Mutwanga et à } \\
\text { Kamango. }\end{array}$ & $\begin{array}{l}\text {-Naissance et résurgence des } \\
\text { groupes armés locaux (URDC, } \\
\text { FRPI...); } \\
\text {-Incendie du Chef-lieu du Secteur } \\
\text { de Ruwenzori par les ADF en } \\
\text { riposte contre les opérations; } \\
\text {-Déplacement massif de la } \\
\text { population du secteur de } \\
\text { Ruwenzori et du Graben des Bashu. }\end{array}$ \\
\hline $\begin{array}{l}\text {. Radi strike } \\
\text { (Coût de foudre) } \\
\text { Le } 20 \text { mars } 2012\end{array}$ & $\begin{array}{l}\text { Désarmer par la force les ADF } \\
\text { et autres groupes locaux (FOLC, } \\
\text { FRPI, MRC, URDC, mayi- } \\
\text { mayi-Eric Kenzo, mayimayi- } \\
\text { Kavawaseli). }\end{array}$ & $\begin{array}{l}\text {-Participation de la délégation } \\
\text { d'Eric Kenzo au dialogue social } \\
\text { d'Ö̈cha, } \\
\text {-Reddition du Colonel David } \\
\text { Lusenge de l'URDC en janvier } \\
2013 \text {; } \\
\text {-Arrestation du Général Kakolele, } \\
\text { Chef du MRC; } \\
\text {-Reddition du Commandant } \\
\text { Kavawaseli). }\end{array}$ & $\begin{array}{l}\text {-Multiplication d'actes ignobles } \\
\text { contre la population civile par les } \\
\text { ADF (enlèvements, kidnappings, } \\
\text { assassinats par décapitation, vols); } \\
\text {-Grande campagne de recrutement } \\
\text { des ADF à l'intérieur comme à } \\
\text { l'extérieur de la RDC; } \\
\text {-Fermeture des axes routiers Mbau- } \\
\text { Kamango et Eringeti-Kainama ; } \\
\text {-Attaques contre les hélicoptères et } \\
\text { les cibles de l'ONU (MONUSCO). }\end{array}$ \\
\hline $\begin{array}{l}\text { 6. Sokola } 1 \\
\text { (Nettoyer } 1) \\
\text { Le } 16 \text { janvier } \\
2015\end{array}$ & $\begin{array}{l}\text {-Sensibiliser les ADF à déposer } \\
\text { les armes sans condition; } \\
\text {-Sensibiliser les congolais ayant } \\
\text { adhéré aux ADF de se } \\
\text { désolidariser d'avec les } \\
\text { Ougandais; } \\
\text {-Libérer les otages des ADF; } \\
\text {-Arrêter le cycle des } \\
\text { enlèvements, des kidnappings, } \\
\text { d'assassinats et toute autre } \\
\text { forme de répression sur la } \\
\text { population congolaise; } \\
\text {-Mettre fin à la présence des } \\
\text { ADF en RDC }\end{array}$ & $\begin{array}{l}\text {-Réouverture de la route Mbau- } \\
\text { Kamango; } \\
\text {-Libération et/ou évasion de } \\
\text { certains otages; } \\
\text {-Reddition du Général dissident } \\
\text { Hilaire Kombi de l'URDC; } \\
\text {-récupération de tous les quartiers } \\
\text { généraux des ADF par les } \\
\text { FARDC; et/ou reddition des } \\
\text {-Capture des réseaux } \\
\text { plusieurs combattants ADF; } \\
\text {-coupure d'approvisionnement des ADF; } \\
\text { d'apblicité et dénonciation du } \\
\text {-Pún plan } \\
\text { phénomène ADF au } \\
\text { international ; des endroits jadis } \\
\text {-Déminage des } \\
\text { occupés par les ADF; } \\
\text {-Fuite de Jamil Mukulu, leader } \\
\text { spirituel des ADF suivi de son } \\
\text { arrestation en Tanzanie en Mai } \\
\text { 2015; } \\
\text {-Découverte des fosses }\end{array}$ & $\begin{array}{l}\text {-Emergence du terrorisme dans la } \\
\text { région de Ruwenzori ; } \\
\text {-Développement de la guérilla par } \\
\text { les ADF; } \\
\text {-Plusieurs assassinats contre la } \\
\text { population civile ; } \\
\text {-Division au sein du leadership } \\
\text { local; } \\
\text {-Arrestation arbitraire des leaders } \\
\text { locaux; } \\
\text {-Division au sein de la société } \\
\text { civile; } \\
\text {-Immigration et installation } \\
\text { désordonnée des populations } \\
\text { d'expression kinyarwanda. } \\
\text { - Assassinats du Colonel Mamadou } \\
\text { Ndala Moustafa et mort inopinée } \\
\text { du Général Jean Lucien Bauma } \\
\text { Ambamba dans des circonstances } \\
\text { jusque-là non illucidées. }\end{array}$ \\
\hline
\end{tabular}




\begin{tabular}{|l|l|l|l|}
\hline & $\begin{array}{l}\text { communes ; } \\
- \text { Capacitation des FARDC à la } \\
\text { guérilla et en matériel de guerre. }\end{array}$ & \\
\hline
\end{tabular}

Comme il ne sera pas facile de revenir sur les six opérations. L'analyse va plus s'intéresser aux deux opérations : Safisha Ruwenzori et Sokola I. La première pause les jalons sur les facteurs de l'échec des toutes les autres qui ont précédé tel que récapitulés dans le tableau 1. La seconde permet de discuter de l'histoire du temps présent et donc de l'actualité sur ses écueils ainsi que ses conséquences sur la population civile.

\section{L'opération Safisha Ruwenzori}

Le caractère géostratégique de la région du Ruwenzori, ses richesses, sa position frontalière, son histoire culturelle favorisent la permanence et la persistance du phénomène ADF. C'est pourquoi, l'opération Ruwenzori prise parmi toutes celles qui y ont été organisées pour la traque des ADF, constitue un modèle type non seulement de la persistance de ces inciviques ougandais mais aussi la preuve de l'existence d'un réseau important impliquant plusieurs acteurs et facteurs qui lient les ADF à leur zone d'influence. L'opération Ruwenzori est officiellement et unilatéralement lancée le 25 juin 2010 à la veille de la célébration du cinquantenaire de l'indépendance de la RDC où le Président J. Kabila devait inviter ses homologues en général et en particulier le Président ougandais Yoweri Kaguta Museveni. Pour un observateur de l'ONU au NordKivu, cette opération «est essentiellement destinée à faire plaisir à Museveni et pour ce faire lui ôter le prétexte de menace imminent d'entrer au Congo alors qu'on s'approche des festivités cinquantenaires de l'indépendance du pays fixées au 30 juin 2010 et des échéances électorales de $2011 »^{5}$.

L'armée congolaise avait indiqué que cette opération devait se poursuivre jusqu'à la «neutralisation complète» ${ }^{6}$ de ces rebelles qui avaient refusé de désarmer malgré la promesse d'un rapatriement en Ouganda, leur pays, assorti d'une garantie d'amnistie. L'on a en même temps signalé la complicité de certains officiers FARDC et UPDF dans un circuit mafieux en collaboration avec les ADF. Ce désordre au sein des FARDC ainsi que la pesanteur externe de l'Ouganda qui exigeait son implication directe dans les combats contre les ADF ne pouvaient pas faciliter une bonne préparation des opérations.Des infiltrations des ADF continuaient à être signalées tant en Ouganda qu'en RDC. L'Ouganda accusa de nouveau le gouvernement de Joseph Kabila et la Monusco de relâcher la pression sur les ADF et de faciliter leur réorganisation. Ce qui fait que l'Ouganda décida de positionner ses troupes sur les hauteurs du Mont Ruwenzori à l'intérieur de la RDC.

A l'issue de la réunion tenue à Lamia le 29 juillet 2009, traitant de cette question, le Mwami Roger Saa-Mbili Bamkoka de la Chefferie des Watalinga écrit que «les Ougandais ont toujours justifié leur présence sur les hauteurs du Mont Ruwenzori, en violation des lois internationales, par les accusations selon lesquelles les Congolais hébergent leurs ennemis à Eringeti, Bovata, Kikingi et ailleurs. Cet argument sert de prétexte presque légitime, pour agresser la $R D C »^{7}$. Rappelons par contre que le Président Ougandais avait déjà déclaré la «défaite » des ADF en avril 2001, à marge de plusieurs interventions militaires de l'UPDF pendant les deux guerres d'agression en 1996-1997 avec l'AFDL et en 1998- 2003 avec le MLC, le RCD et RCD-KML. Dans un tract intercepté par la population d'Eringeti, les rebelles ADF accusaient les militaires aussi bien congolais qu'ougandais d'être impliqués dans le circuit de trafic de l'or, du bois et du café en échange de matériels de guerre. Et dénonçaient ce qu'ils qualifiaient d'acharnement contre eux. Les ADF résistèrent à l'offensive des FARDC. Après une certaine accalmie, entre 2005 et 2007 l'exploitation du bois et le trafic de l'or prirent de l'ampleur au point de bloquer les opérations de traque contre ces rebelles ougandais des ADF. Ce qui leur a facilité de récupérer leurs anciennes positions et d'étendre leur zone d'influence. C'est dans cette foulée qu'on peut situer l'attaque du 25 avril 2010 contre le Centre de Brassage et de Recyclage (CBR) de Nyaleke, à 7 km de la ville de Beni, dans laquelle une coalition ADF-Mayi Mayi avait massacré femmes et enfants appartenant aux familles militaires. Ceci obligea le gouvernement de la RDC à relancer les opérations contre les ADF. C'est l'opération « Safisha Ruwenzori ».

Selon le Général Vainqueur Mayala ${ }^{8}$, cette offensive lancée le 27 juin 2010 avait permis aux troupes congolaises de récupérer Mughalika le quartier général des ADF, dans le Graben, en chefferie des Bashu. Ce camp permettait aux ADF de se nouer d'importantes lignes logistiques notamment avec la ville commerciale de Butembo. Dans leur riposte, dans la nuit du 27 au 28 juin, soit la veille de la célébration du cinquantenaire de l'indépendance de la RDC, le 30 juin 2010, les ADF attaquèrent et incendièrent le bâtiment du bureau

\footnotetext{
${ }^{5}$ Interview nous accordée par un haut fonctionnaire de l'ONU (Monusco) à Goma, le 14 juillet 2010.

${ }^{6}$ International Crisis Group, $L$ 'Est du Congo : la rébellion perdue des $A D F-N A L U$, Briefing Afrique n ${ }^{\circ} 93$, Nairobi/Bruxelles, 19 Décembre 2012.

${ }^{7}$ Résolution de la rencontre mixte autorités du district de Bundibugyo et du territoire de Beni tenue à Bundibugyo en date du 15 Décembre 2006

${ }^{8}$ Jaribu Muliwavyo, «Analyse des politiques d'éradication de la rébellion des ADF-NALU dans le Ruwenzori », in CSSAP, N5, Goma, août 2016, pp. 16-36. 
administratif ainsi que la résidence officielle du Chef de secteur Ruwenzori à Mutwanga ${ }^{9}$. Ils y furent 9 morts dont 7 civils et 2 policiers. Cette attaque provoqua un déplacement de 100.000 vers Bulongo et Beni. Le 31 juillet à la veille de la visite à Beni et Mutwanga du Ministre congolais de la Défense, Mwando Simba des tracts signés par les ADF, le 23 juillet 2010, furent ramassés. Ceux-ci dénonçaient les exactions contre les ADF par les FARDC. Ces $\operatorname{tracts}^{10}$ accusaient, comme ceux d'Eringeti, certains officiels de ne pas respecter «les arrangements » qu'ils auraient conclus avec elles. Ces accords leur autorisaient, selon les ADF, le séjour en RDC à condition de ne pas s'ingérer dans les affaires intérieures congolaises. L'écho de ces tracts fit des tollés en Ouganda qui décida de réorganiser quelques rebelles pour se mettre aux aguets. Deux lettres manuscrites furent découvertes par les services spécialisés de la RDC au poste de Kasindi. L'on pouvait lire dans leur rapport : «il se répand en effet en Territoire de Beni, des rumeurs selon lesquelles un mouvement subversif susceptible de déstabiliser la contrée voire même la Province ou le pays entier se prépare en Ouganda. Il s'agit d'un certain mouvement rebelle entretenu par une coalition d'ex-combattants issus du mouvement MaïMaï/Ruwenzori et de l'ex-APC. Les précités ont adressé l'une des lettres au ministre ougandais de la défense et l'autre à un officier de renseignement ougandais à Mpondwe. Dans ces deux lettres, ce nouveau mouvement rebelle, baptisé Forces Ecuméniques pour la Libération du Congo FOLC en sigle, sollicite du Gouvernement ougandais un appui en armes, en hommes, en vivres, en médicaments, et un centre d'entraînement ${ }^{11}$. Heureusement, lors de la prestation de serment du Président Joseph Kabila pour son deuxième mandat en 2011, les relations entre la RDC et l'Ouganda s'étaient renouées. Dans l'autre camp, entre novembre et décembre 2011, les ADF conclurent une série d'alliances à la fois avec les FDLR ${ }^{12}$ et le FRPI. Ces alliances permirent à un groupe de combattants ADF de rejoindre le Front de Résistance Patriotique de l'Ituri (FRPI) dans la région de Tchabi et Boga peut-être pour se constituer des zones de retrait en cas d'attaque. En cette même période, le Président Kabila ordonna le déploiement d'anciens membres du CNDP dans la région de Beni pour «sécuriser» le scrutin contre une éventuelle attaque des ADF ; l'on ne saura pas dire à faveur de sa réélection. Tous ce qu'il faut dire, c'est que, les anciens rebelles du CNDP avaient à leur tête un commandant originaire de la région qui, pour y avoir opéré notamment au sein des FAZ, dans l'APC et même sous son propre Mouvement Révolutionnaire Congolais (MRC) ${ }^{13}$, jouissait d'une confiance du chef de l'Etat congolais pour faciliter les bons offices auprès des ADF. Dans cette effervescence électorale, «cet officier militaire adhéra au PPRD, parti du Chef de l'Etat $»^{14}$. Accusé d'être de mèche tantôt avec ses anciens compagnons du CNDP qui venaient de faire défection pour les M23, tantôt avec les ADF, il fut arrêté puis ramené manu-militari à Kinshasa. Il sera libéré quelques mois plus tard sans aucun procès.

Dans son adresse à la population du Nord-Kivu, le $1^{\text {er }}$ janvier 2012 à l'Hôtel Ihusi à Goma, Joseph Kabila informa les représentants des forces vives de la suspension des opérations militaires au Nord-Kivu. Cet arrêt d'opérations militaires permit aux ADF de reprendre leurs anciens campements de Chuchubo et Makembi, le $1^{\text {er }}$ juillet 2011 ainsi que le village Bilimani le 29 juillet 2011. Les ADF consolidèrent leurs blockhaus à l'est d'Oïcha. Les rebelles repoussèrent l'armée congolaise mêlée aux soldats ougandais, et à la MONUSCO à Nadwi, Makoyova I et II, dans le Groupement Banande-Kainama. De l'autre côté de la frontière en Ouganda, suite aux multiples attaques des rebelles contre les humanitaires, beaucoup d'agences d'aide internationale s'étaient retirées du district de Bundibugyo par crainte de la détérioration de la situation sécuritaire ${ }^{15}$. Ces mêmes pratiques vont s'observer en RDC avec l'enlèvement des agents de Médecins Sans Frontière (MSF) lors des dernières attaques de Kamango en novembre 2013.Une carte d'identité de l'un de ces quatre agents avait été d'ailleurs retrouvée à Medina pendant l'opération Sokola 1. Cette carte avait permis aux services de sécurité de couper cours aux spéculations sur l'identité des kidnappeurs dans le territoire de Beni. Désormais l'armée ainsi que l'administration locale savaient orienter les renseignements sur la libération des otages dont le nombre s'accroissait rapidement.

\footnotetext{
${ }^{9}$ Visite du lieu des événements et participation aux cérémonies funéraires de 9 victimes à Mutwanga le 28 juin 2010.

${ }^{10}$ Mémorandum adressé au Gouverneur de la Province du Nord-Kivu par la notabilité de Mutwanga sollicitant l'installation d'une base de la MONUSCO pour sécuriser la population civile du secteur de Ruwenzori, Mutwanga, le 14 juillet 2010.

${ }^{11}$ Assemblée provinciale du Nord-Kivu, synthèse des rapports des vacances parlementaires du 07 octobre 2013, in « Annales parlementaires de l'Assemblée provinciale du Nord-Kivu ».

${ }^{12}$ Entretien nous accordé par Abdoul, ancien élément des ADF, détenu à la prison centrale de Kangbwayi/ Beni, le 22-08-2014.

${ }^{13}$ Entretien nous accordé par le commandant B.K, autoproclamé Général des FARDC, Beni, le 5 janvier 2013.

${ }^{14}$ A la lumière de l'article 188 de la constitution de la RDC, les Forces Armées sont républicaines. Elles sont au service de la nation toute entière. Nul ne peut, sous peine de haute trahison, les détourner à ses fins propres. Elles sont apolitiques et soumises à l'autorité civile. A ce titre, les FARDC ainsi que la Police Nationale Congolaise (PNC) ne peuvent pas appartenir à un parti politique.

${ }^{15}$ Rapport for Refugee Law Project and Save the children, child Protection in the context of displacement : Ntoroko country and Bundibugyo District, Kampala, d2C ; 2004 
Tableau 2. Effectifs d'enlèvements de juin 2010 à août 2013

\begin{tabular}{|c|c|c|c|c|c|}
\hline Année & Groupement & Village (lieu d'enlèvements) & $\mathbf{H}$ & $\mathbf{F}$ & Total \\
\hline 2010 & & Abialose & 21 & 3 & 24 \\
\hline \multicolumn{3}{|l|}{ Sous total } & 21 & 3 & 24 \\
\hline \multirow[t]{3}{*}{2011} & Bambuba Kisiki & Tungudu-Kokola & 16 & 1 & 17 \\
\hline & Batangi-Mbau & Bilimani-Tedeu & 14 & 6 & 20 \\
\hline & Cité d'Oicha & Q. Oicha 1er, Cel. Hopital & 1 & 0 & 1 \\
\hline \multicolumn{3}{|l|}{ Sous total } & 31 & 7 & 38 \\
\hline \multirow[t]{2}{*}{2012} & Bambuba-Kisiki & Kamakombu-Abialose-Tungudu & 47 & 7 & 54 \\
\hline & Batangi-Mbau & Mbau-Centre, Bilimani, Tedeu, Totolito & 39 & 22 & 61 \\
\hline \multicolumn{3}{|l|}{ Sous total } & 76 & 39 & 115 \\
\hline \multirow[t]{2}{*}{2013} & Bambuba-Kisiki & Kamakombu-Silimbamba & 165 & 120 & 285 \\
\hline & Batangi-Mbau & $\begin{array}{l}\text { Mbau III, Mamundioma, Bilimani, Tedeu, } \\
\text { Totolito, PK11-PK28 Route Kamango }\end{array}$ & 299 & 152 & 451 \\
\hline \multicolumn{3}{|l|}{ Sous total } & 464 & 272 & 736 \\
\hline 2013 & Secteur Watalinga & Bahumu, Bawisa & 203 & 35 & 238 \\
\hline \multicolumn{3}{|l|}{ Sous total } & 203 & 35 & 238 \\
\hline 2013 & Secteur Ruwenzori & Banyangala. & 31 & 9 & 40 \\
\hline \multicolumn{3}{|l|}{ Sous total } & 31 & 9 & 40 \\
\hline \multicolumn{3}{|l|}{ Total général } & 826 & 365 & 1191 \\
\hline
\end{tabular}

Source : Constitué sur base des messages officiels du service Okapi du Territoire de Beni de 2010 à 2013.

Ce tableau ne concerne que les enlèvements considérés officiellement comme ayant été effectués par les ADF. En plus de ce cas d'enlèvements répertoriés, d'autres individus avaient seulement été exécutés par leurs ravisseurs. A Oicha par exemple, le seul cas identifié et le tout premier au chef-lieu du territoire de Beni a été celui du Docteur Paluku Mukongoma, Médecin Directeur de l'hôpital général d'Oîcha, enlévé la journée du $1^{\text {èr }}$ juillet 2011, alors qu'il était en plein service. Il sera suivi des trois Pères assomptionnistes, enlevés le vendredi 19 octobre 2012 à la paroisse de Mbau, dont les pères Anselme Wasukundi, Jean-Pierre Ndulani et Edmond Kisughu. C'est dans la même série d'enlèvements qu'on peut citer le cas de quatre agents de MSF pendant l'attaque de Kamango, le 11 Juillet 2013.Ces enlèvements obligèrent les autorités congolaises à planifier une nouvelle opération militaire contre les ADF.

\section{L'opération Sokola 1}

Une nouvelle planification d'opérations de traque contre les ADF baptisés SOKOLA I reprit le vendredi 22 novembre 2013, à Goma. Au centre de la réunion tripartite, étaient présents les Chefs des EtatsMajors, Généraux Didier Etumba pour les FARDC, Aronda Nyakayirima pour l'UPDF et Alberto Santos Cruz pour la MONUSCO. A l'issue de cette réunion, les officiels congolais déclarèrent que « la traque des rebelles va être l'affaire des seules FARDC avant de signifier que les assises avaient pour unique objectif de prévenir des dégâts collatéraux qui affecteraient l'Ouganda ${ }^{16}$. C'est dans une déclaration de presse que le Colonel Mustafa Mamadou Ndala donne l'objectif de cette opération. Selon lui : « l'ordre était donné à l'Armée pour libérer nos pères, nos mères, nos frères et nos sœurs qui ont été kidnappés par les ADF. Qu'ils soient morts nous devons retrouver leurs tombes et même s'ils ont été jetés dans l'eau (rivière Semuliki) nous devons retrouver le poisson qui les a avalé » ${ }^{17}$.

Les ADF étaient déjà avisés du déluge qui se préparait contre eux. Elles développèrent des stratégies de protection. Les forêts du PNVi, difficiles d'accès les avaient habitué et permis de se protéger des différentes attaques. Le 6 janvier 2014, les FARDC lancent l'opération Sokolal contre les ADF. L'opération est menée avec un appui logistique limité de la MONUSCO, y compris une mission d'évacuation sanitaire à Goma. Les soldats de la Brigade d'intervention de la MONUSCO furent basés à Mavivi et à Kamango mais, ne participaient pas à des combats terrestres ; sauf le programme «Ulinzi bora ${ }^{18}$ pour la sécurisation de la ville de Beni. Une stratégie opérationnelle de lutte contre l'insécurité à Beni (SOLIB) fut mise sur pied avec mission de renforcer la PNC en formation, et en logistique. Elle mit ensemble les éléments de la PNC et ceux de l'EUPOL (Mission Européenne de Police). Quelques officiers ougandais s'étaient établis à Beni pour le suivi et l'évaluation de l'opération militaire. Le front était organisé sur trois axes, selon l'ordre de bataille (OB) consulté à l'Etat-major des opérations. ${ }^{19} \mathrm{Ce}$ sont :

\footnotetext{
${ }^{16}$ Communiqué final de la tripartite RDC-Ouganda-Monusco pour les opérations de traque contre les rebelles Ougandais AFD-NALU, sur Radio Okapi, Goma, le 23 novembre 2013.

${ }^{17}$ Propos du Colonel Mustafa Mamadou Ndala, le 28 Décembre 2013.

${ }^{18}$ Entretien nous accordé par Alasan Samake, Commandant de la Police MONUSCO/Beni, le 22 août 2014.

${ }^{19}$ Entretien nous accordé successivement par : - K.M, Major des FARDC à Mamundyoma, le 09 août 2014. - A.M, Général de Brigade des FARDC, Beni, le 02 Avril 2015
} 
- l'axe Mbau- Kamango en passant par Totolito, Mamundyoma, camp Kabila, camp Maître, Mukakati avec un détachement qui déborde vers Medina en empruntant les lisières des rivières Lese, Bango et Abianlose sur la rive gauche de la Semuliki ;

- l'axe Tenambo-Bilimani-Kasuku-Mapobu-Silimbamba pour sortir vers Mamundyoma à jonction avec la première troupe ;

- l'axe Eringeti-Nadui- Saasitasaa-Kazaroho-Mokongo ya koba-Medina avec un détachement vers Kwele et le ratissage des bords de la Semuliki pour empêcher la traversée des ADF vers la rive droite pour le Ruwenzori.

L'opération aurait fait des centaines de victimes, principalement parmi les militaires, mais aucune vérification indépendante des chiffres n'a été réalisée. Toutefois, les officiers des FARDC avaient dit au groupe d'experts de l'ONU qu'au 7 mai, les FARDC avaient perdu 217 soldats morts au combat et recensé 461 blessés, parmi lesquels figurait un certain nombre de victimes de tirs fratricides et que 531 combattants ADF avaient été tués depuis le début de l'opération ${ }^{20}$. Dans une séance de travail, tenue à Goma, le 16 août 2016 entre les membres du Conseil Supérieur Militaire de la RDC avec les députés provinciaux du Nord-Kivu, le Général Didier Etumba Longila confirmait que : «les hommes politiques ne devaient pas accuser les forces armées d'être auteures des massacres à Beni. Les forces armées ont perdu pour ramener la paix en Beni. Pendant les dix derniers jours de combat intense qui nous avaient permis de mettre un terme à la guerre du M23 à Rutshuru, les FARDC avait perdu 200 hommes. Par contre, pour prendre Medina, le quartier général des ADF, où résidait le Chef rebelle Jamil Mukulu, nous nous sommes battus contre l'ennemi pendant trois jours sans interruption et nous avons perdu 148 hommes de troupe. Ce qui atteste que la guerre contre les ADF exige plus d'efforts que celle menée contre les M23 en $2013 »^{21}$.

Un de «nos enquêtés ${ }^{22}$ affirma que certains dirigeants de la rébellion s'étaient retranchés dans la ville de Beni pour échapper aux attaques des FARDC et que d'autres s'étaient dirigés vers le Nord-Est, dans la région d'Abia et Boga; ainsi que dans la forêt de Mambasa en Ituri.En avril 2015, un porte-parole du gouvernement ougandais affirma que Jamil Mukulu, chef des ADF inscrit sur la liste noire des criminels recherché par l'ONU, avait quitté la RDC. « D'autres sources ${ }^{23}$ informaient qu'il se serait retiré vers le Soudan et continuait à donner des ordres à ses hommes au moyen des radios Motorola. Son arrestation à Kagezi, à la frontière entre la Tanzanie et le Kenya la nuit du 21 au 22 avril 2015, n'a été annoncée qu'en mai 2015 par le porte-parole du Gouvernement Ougandais, Ofwono Opondo. D'autres sources indiquent qu'en réalité pour s'évader, «Jamil Mukulu avait emprunté l'axe Eringeti-Bunia où il a pris un avion qui l'amena à Goma d'où il est parti avant $q u$ 'il ne se retrouve en Tanzanie via le Rwanda ${ }^{24}$. Il fut expulsé vers l'Ouganda, le 13 juin 2015.Cette arrestation aurait bien sûr affecté le mouvement sans pour autant déclencher la fin de l'activisme des ADF. Les enlèvements, les assassinats et autres pratiques terroristes ont continué à être signalés dans la région.

L'arrestation de Jamili Mukulu serait le résultat de la collaboration entre les services spécialisés de la Grande Bretagne et de la Tanzanie. Certains de nos enquêtés pensent que c'est pour dénoncer cette incarcération que les ADF avaient multiplié des attaques contre les casques bleues. C'est à cette même période qu'un contingent de casques bleus tanzaniens de la Brigade d'Intervention de la MONUSCO va tomber dans une embuscade, le 5 mai 2015, sur la route Beni-Oïcha. Cet acte ignoble fit deux morts, 13 blessées, 4 disparus et un véhicule incendié. Toutefois, « un rapport des experts du conseil de sécurité de l'ONU » ${ }^{25}$ accuse plutôt les FARDC pour cette attaque du convoi de la MONUSCO. Tel qu'indique la figure ci-dessous, les tronçons qui traversent la zone habituellement sous influence des ADF furent de nouveau déclarés « zone rouge » et donc à haut risque pour les usagers. Ce qui fait douter de l'accomplissement et de la réussite des objectifs de départ de l'opération Sokola 1.

\footnotetext{
${ }^{20}$ Rapport à mi-parcours du groupe d'experts de l'ONU sur la RDC, New-York, le 19 juin 2014

${ }^{21}$ Propos du Général d'armée Didier Etumba Longila, Chef d'Etat-major Général des FARDC. Musée de Himbi/Goma, le 16 Août 2016.

${ }^{22}$ Entretien nous accordé par le général-Major Jean Lucien Bauma Ambamba le 21 mars 2014 à l'hôtel Albertine de Beni quelques jours avant sa mort.

${ }^{23}$ Entretien nous accordé par Kambale Ushindi ; un élément ADF-NALU capturé par la population civile d'Oïcha et présenté au Bureau de l'Administrateur du territoire de Beni à Oïcha, le 7 août 2014

${ }^{24}$ Rafiki, Journal Indépendant d'Informations Générales, ${ }^{\circ}$ 19-Du 5 mai au 5 Juin 2016.

${ }^{25}$ www.rfi.fr, lu le 5 Février 2016 


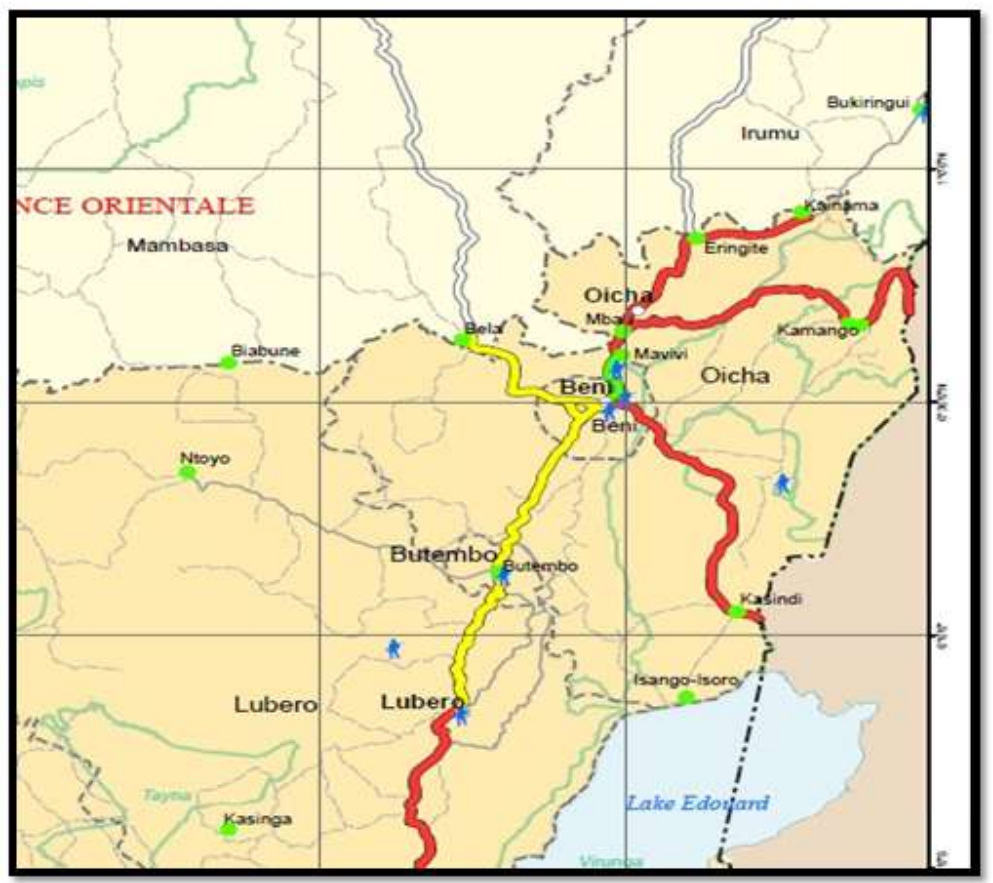

Figure1. De la morphologie sécuritaire de la région du Ruwenzori après l'arrestation de Jamil Mukulu Source : Rapport de monitoring de la section sécurité MONUSCO/Nord-Kivu, juin 2015

Légende :

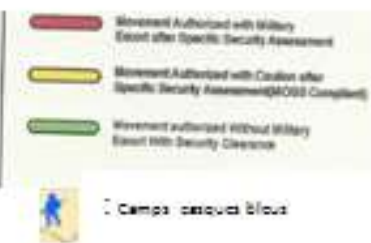

Quatre raisons justifient la résurgence des exactions des ADF contre la population civile ainsi que la régression à puissance des FARDC.

1. A la mort du Général Jean Lucien Bahuma Ambamba, les hommes de troupes étaient démotivés. Les militaires FARDC qui étaient à la première ligne des combats n'arrivaient pas à digérer cette mort à quelques mois de celle du Colonel Mamadou Ndala. Tous les deux sont morts dans des circonstances obscures ;

2. Après la mort du Général Jean Lucien Bahuma Ambamba, les militaires engagés dans l'opération firent trois mois sans recevoir leur solde. « Des épouses militaires du camp OZACAF manifestèrent devant la mairie de Beni pour exiger le paiement de la solde de leurs époux engagés dans les zones des combats contre les ADF. Une soixantaine des femmes des militaires du $808^{\text {ème }}$ régiment avaient marché, lundi 29 septembre 2014, à partir du camp de Mukakira/Ö̈cha et tentèrent de joindre Beni sur un tronçon d'environ $30 \mathrm{~km}$. Elles furent empêchées et arrêtées à Mbau, à $7 \mathrm{~km}$, par la $\mathrm{PNC}{ }^{26}$;

3. Les militaires de rang attendaient la désignation d'un nouveau Commandant des opérations en remplacement du Général Bahuma ; celui qui pouvait rassurer toutes les unités. A leur grande surprise, selon un Colonel, «c'est le Commandant de la 31ème Brigade, contesté voire suspecté par la population suite aux comportements de ses hommes de troupes (tracasseries, assassinats présumés des civils) et du soupçon dans l'assassinat du Colonel Mamadou Ndala, qui prit les reines de l'opération Sokola 1, mais également de la zone opérationnelle grand-nord allant d'Eringeti à Kanyabayonga » ${ }^{27}$. De son exil à l'étranger, le 24 Octobre 2014, un ancien Ministre des Affaires Etrangères de Joseph Kabila déclara que : «cet officier était en même temps commandant des ADF et qu'il n'était pas le bienvenu à la tête des FARDC dans cette zone opérationnelle ${ }^{28}$;

\footnotetext{
${ }^{26}$ www.radiookapi.net lu le 29 Septembre 2014.

${ }^{27}$ Entretien nous accordé par le Chef de la section de communication de la MONUSCO/Beni, Mavivi, le 18 février 2015.

${ }^{28}$ Intervention d'Antipas Mbusa Nyamwisi, Député national élu de Butembo, sur la FRI, vendredi 24 octobre 2014. Ce dernier avait réitéré les mêmes propos sur la Voix des Africains du Canada (VAC), le 2 février 2015 en accusant le régime du Président Joseph Kabila d'être à la base des massacres des civiles dans la région de Beni.
} 
4. la méfiance du gouvernement Congolais vis-à-vis de la MONUSCO. En effet, selon les déclarations de Lambert Mende Omalanga, Ministre de l'Information et porte-parole du gouvernement, les FARDC étaient à elles-mêmes capables de traquer les $\mathrm{ADF}$. «les FARDC n'ont pas besoin d'une quelconque aide. Je vous donne le bilan des opérations Sokola à Beni. Plus de 200 éléments tués du côté des ADF. Les pertes sont insignifiantes du côté FARDC. Nous avons récupéré un important stock d'armes et des munitions par rapport à d'autres zones récupérées auparavant, ce qui nous laisse croire que Saasita-Saa était la dernière place forte de ces forces négatives... les combats ne s'arrêteront que lorsque le dernier ADF se trouvant en RDC aura été désarmé ou neutralisé ou aura quitté le territoire congolais. Avec ça, nous n'avons pas besoin d'une quelconque force étrangère qui mettra notre souveraineté en péril, surtout avec la montée en puissance des FARDC ${ }^{29}$. En comparant ce bilan donné par Lambert Mende Omalanga avec celui des experts de l'ONU précédemment référé, il y a lieu de croire à la véracité de ces renseignements même si le Ministre semble avoir dérobé au principe diplomatique du devoir de réserve.

Cette indiscipline aussi bien communicationnelle que militaire eut des conséquences néfastes sur la bonne marche de l'opération Sokola 1 notamment :

- Les militaires au front furent désormais dans une zone opérationnelle où ils n'inspirent pas confiance à la population. Ils sont donc dépourvus d'informations nécessaires sur l'ennemi ;

- Les unités de lutte furent relevées de la zone de combat. Un colonel stigmatise : « le fait que ces derniers avaient refusé de recevoir des ordres d'un officier qui n'était pas de leur unité ; les 41ème et 42ème bataillons des commandos des Unités de Réaction Rapide (URR) adapté à la guerre asymétrique, jadis commandés par le Colonel Mamadou Ndala Mustafa ». Ce qui entraina un rélachement au niveau du front ainsi que la réorganisation des quelques camps des $\mathrm{ADF}$.

Cette cacophonie au sein des FARDC fut profitable aux ADF. Elles se reconstituèrent en érigeant de nouveaux campements dont Isa, Banuku vers Medina, leur réinstallation à Mughalika et la prolifération des nouvelles bases autour de Supa-Kalau-Lisasa dans les périphéries du PNVi où ils ont repris et relancé le recrutement pour la formation des nouveaux combattants ADF. Les massacres prirent de l'ampleur.

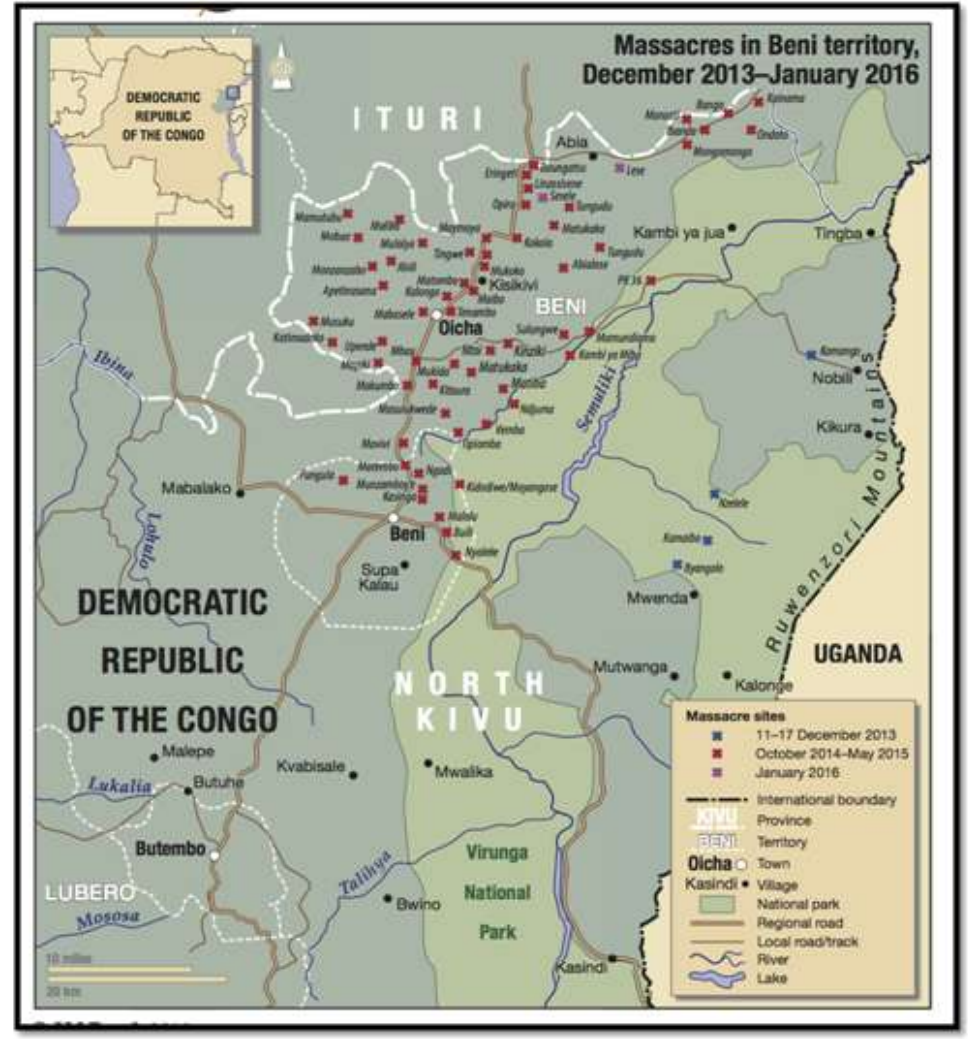

Figure 2. Des massacres des civils de 2013 à 2016

Figure 12. Cartographie des massacres dans la région du Ruwenzori de 2013 à 2016.

Source :- Groupe d'Etude sur le Congo, mars 2016.

- C'est nous qui adaptons pour août 2016

${ }^{29}$ Propos de Lambert Mende Omalanga, dans une conférence de Presse tenue à Kinshasa et rélayée par la presse locale, le 24 juin 2014.

DOI: 10.9790/0661-1901015664 www.iosrjournals.org $\quad 63 \mid$ Page


Lancée le samedi 14 mai 2016, l'opération «Usalama » avec comme objectif « d'éradiquer totalement les terroristes ADF qui ne cessaient d'endeuiller la population du territoire de Beni sur qui ils commettaient des exactions meurtrières $»^{30}$. L'armée congolaise appuyée par les forces de la MONUSCO, présente un bilan à miparcours de cette opération. Selon le Lieutenant Mak Hazukay, porte-parole militaire du secteur opérationnel grand nord et des opérations Sokola I: «les FARDC avec la MONUSCO ont tué 37 combattants ADF et a capturé 15 autres. L'opération a permis la récupération d'armes et munitions : Treize AK 47, deux RPG7, une caissette de munitions PKM, deux bombes artisanales, dix besaces et une batterie ${ }^{31}$. Ce bilan venté par Joseph Kabila lors de son meeting à Kasindi, jeudi 4 août 2016 dans lequel il promet la paix a été perturbé par un nouveau massacre, la nuit de samedi 13 à dimanche 14 août 2016 à Mbelu en pleine ville de Beni, avant que le Chef de l'Etat ne clôture son séjour dans la région de Beni.

En effet, en dépit de l'opération Sokola 1, un contexte sécuritaire anormal s'observe à travers plusieurs carnages. Beni devient ainsi une véritable boucherie humaine. Il s'y développe notamment :

$\ll$

- l'extension de la menace terroriste sur la zone en conflit ;

- le nombre de personnes kidnappées, en majorité des civils, continue à galoper ;

- depuis le massacre du 11 au 17 décembre 2013 dans la région de Biangolo à nos jours, l'on compte environ 1113 personnes égorgées à la hache et à la machette;

- des bombes larguées et ou déposées dans des lieux populaires ont fait des victimes en pleine ville de Beni ;

- l'arrêt momentané d'activités quotidiennes de la population : impossibilité d'accès aux champs, pas de scolarité, des centres de santé détruits ;

- une crise humanitaire devenue continue $»^{32}$.

\section{Conclusion}

Cette discussion sur les opérations militaire visant à éradiquer le phénomène ADF dans le territoire de Beni, présente les forces et les ratés des stratégies d'un long processus de pacification de la région du Ruwenzori. Ces stratégies se situent au déversoir des intérêts géographiques et géostratégiques qui en constituent aujourd'hui à la fois l'épine dorsale des conséquences néfastes vécues en ce lieu. De la géopolitique de la région, des intérêts géostratégiques de l'Ouganda et du Zaïre à l'époque et de la RDC actuellement, la diplomatie par rébellions interposées qui a jalonné les relations entre les deux Etats concourent à l'émergence de cette rébellion. La crise du leadership régional et local renforcée par la culture du silence et l'idéologie de la mort développées par ces rebelles leur ont permis de se constituer un réseau impliquant les acteurs de tout bord, les uns opposés et/ou alliés, internes et/ou externes. C'est par exemple les renforcements des FARDC en équipement militaire, leur spécialisation par rapport à la zone des combats (les lacs Edouard, et Albert ainsi que la rivière Semuliki, les parcs aux forêts et le massif montagneux), la redéfinition des relations entre les FARDC et la population civile, la levée de confusion sur l'utilisation des tenues des FARDC par les ADF, la relance et la rationalisation du programme de démobilisation, l'implication des leaders locaux dans l'appropriation des opérations par la population locale pour la destruction du réseau des ADF et l'évaluation citoyenne et/ou participative de l'opération Sokola 1.Ces mesures permettront de renforcer l'autorité étatique en vue de la consolidation d'une paix durable au profit des peuples de la région et de Beni en particulier. Ce qui ferait la fierté et le prestige de l'Etat congolais dans ce coin.

\footnotetext{
${ }^{30}$ Entretien nous accordé par le Lieutenant Mak Hazukay, le 3 Août 2016 à l'hôtel Vyaka à Beni.

${ }^{31}$ Entretien nous accordé par le Lieutenant Mak Hazukay, le 3 Août 2016 à l’hôtel Vyaka à Beni.

32 - Rapports d'activité de l'Association Africaine des Droits de l'Homme (ASADHO/Beni), de novembre 2014 à août 2015.

- Human Rights Watch, RD Congo : des attaques menées par des rebelles ont fait plusieurs dizaines de morts, Goma, 16 décembre 2014.

- Assemblée Nationale, Rapport de la mission d'information et de réconfort auprès des populations de la ville de Beni et des agglomérations du territoire de Beni victimes des tueries du 02 au 21 octobre 2014. 